\title{
Performance Analysis of Digital Wireless Networks with ARQ Schemes
}

\author{
Wuyi Yue ${ }^{1}$ and Shunfu $\mathrm{Jin}^{2}$ \\ ${ }^{1}$ Department of Information Science and Systems Engineering \\ Konan University, Kobe 658-8501 Japan \\ yue@konan-u.ac.jp \\ ${ }^{2}$ College of Information Science and Engineering \\ Yanshan University, Qinhuangdao 066004 China \\ jsf@ysu.edu.cn
}

\begin{abstract}
In this paper, we present a method to analyze the performance of high-reliability Internet systems in wireless environments with Automatic Repeat ReQuest (ARQ) schemes. Considering the setting up procedure of a data link in wireless networks, we build a Geom/G/1 queue model with a setup strategy to characterize the system operation, and analyze the probability distribution of the system to obtain the performance measures. Numerical results are given to evaluate the performance of different ARQ schemes in terms of the response time and the utility, and to show influence of the delay of the setup procedure and the round-trip-time on the system performance.
\end{abstract}

Keywords: ARQ, setup, wireless networks, performance analysis.

\section{Introduction}

Wireless networks represent a challenging and ever growing research area, supports for Internet services with excellent reliability in wireless networks are emerging requirements [1. As a close-loop error control technique, Automatic Repeat ReQuest (ARQ) schemes have been shown to be very efficient and successful in wireless communication environments [2], 3]. In these systems, the interactions among the different network layers are very complex and their effects on the overall performance are not easy to accurately identify.

Queueing theory and Markov chains are used for the performance evaluation of ARQ schemes. 4] has indicated that it would be more accurate and efficient to use discrete-time queueing models than continuous-time queueing models when analyzing and designing digital transmitting systems.

The classical discrete-time queuing analyses have been presented in [4], [5]. Analysis of a discrete-time queueing model with setup strategy can be found in [6]. A Geom/G/1 queue model with a setup/close-delay/close-down strategy was built and analyzed by using an imbedded Markov chain in 77. The same model using the approach of factorization principles with general vacations was analyzed in 8]. 
Extensive researches of advanced ARQ schemes as well as some performance analyses based on classical ARQ schemes have been conducted in [2], [3] and [9]. However, some simplifying assumptions considered in these studies do not hold in practice. For example: the setting up procedure of a data link was neglected and the round-trip-time was omitted. It is important to present influence of the delay of the setting up procedure and the round-trip-time on the system performance in such wireless communication networks.

To give actual system models and high quality performance evaluation, we relax these simplifications in this paper. Considering a memoryless session initiated by users, and taking into account the delay of the setting up procedure of a data link and the round-trip-time, we build a Geom/G/1 queue model with a setup strategy to characterize the system operation. Then we analyze the performance of the system with different ARQ schemes in terms of the response time of data frames and the utility ratio of the system. Based on numerical results, we evaluate the system performance and show influence of the delay of the setting up procedure and the round-trip-time on the system performance.

\section{System Model}

The system consists of a paired transmitter and receiver. When two adjacent users need to communicate each other, a data link must be set up by exchanging some control signals. When the data transmission finishes, the data link should be released. The system works as follows. And this process will be repeated.

(1) When a data frame arrives in the system, a setup period called $U$ will be started, where $U$ corresponds to a time period for setting up a data link.

(2) After the setup period $U$ finishes, a busy period called $\Theta$ will begin. Here we define the busy period $\Theta$ to be a time period in which data frames are transmitted continuously until the buffer of the transmitter becomes empty.

(3) When there are no any data frames in the buffer of the transmitter to be transmitted, the data link will be released and the system will enter an idle period called $I$. A data frame arriving during $I$ will make the system to enter a new setup period $U$ again.

The time axis is divided into slots of fixed length. The length of a slot is defined as a time period from the instant that the first bit of a data frame is sent out to the instant that the last bit of the data frame is sent out.

A Geom/G/1 queue model with a setup strategy is presented to analyze the probability behavior of such a system. Considering the memoryless character of the user's initiated session, we assume that the input follows a Bernoulli process. Namely, the number of data frames arriving in a slot is assumed to be 1 with the probability $\lambda$, and 0 with the probability $1-\lambda$.

We define a transmission period $B$ called delivery delay as being the time period taken to successfully transmit a data frame. Namely, that is the time period from the instant for the first transmission of a data frame to the instant for the departure of the data frame from the transmitter buffer. 
There are three kinds of basic ARQ schemes: Stop-and-Wait ARQ scheme, Go-Back-N ARQ scheme and Selective-Repeat ARQ scheme.

For all kinds of the ARQ schemes, the actual delivery of a data frame only occurs after the correct reception of all data frames with lower identifier, so we can assume that data frames arriving in the buffer with an infinite capacity are transmitted using a common data link, one by one, in a FIFO discipline.

\section{Performance Analysis}

Setup period $U$ and transmission period $B$ are assumed to be independent discrete-time random variables in slots, and are assumed to be generally distributed. The Probability Generation Function (P.G.F.) $U(z)$ and the average $E[U]$ of $U$, as well as the P.G.F. $B(z)$ and the average $E[B]$ of $B$ are given as:

$$
\begin{aligned}
& u_{k}=P\{U=k, k \geq 1\}, \quad U(z)=\sum_{k=1}^{\infty} u_{k} z^{k}, \quad E[U]=\sum_{k=1}^{\infty} k u_{k}, \\
& b_{k}=P\{B=k, k \geq 1\}, \quad B(z)=\sum_{k=1}^{\infty} b_{k} z^{k}, \quad E[B]=\sum_{k=1}^{\infty} k b_{k} .
\end{aligned}
$$

We present the performance analysis of the system in a state of equilibrium, namely $\rho=\lambda E[B]<1$.

\subsection{Queueing Length and Waiting Time}

In a late-arrival system with immediate access, we assume that data frame arrivals and departures occur only at the boundaries of slots. The arrival of a data frame during the slot $t$ is assumed to occur at the instant $t^{-}$, and the departure of a data frame during the slot $t$ is assumed to occur at the instant $t$.

Let $Q_{n}=Q\left(t_{n}^{+}\right)$be the number of data frames in the system immediately after the $n$th data frame departed. Then $\left\{Q_{n}, n \geq 1\right\}$ forms an imbedded Markov chain. We define the state of the system by the number $Q$ of the data frames in the system at the imbedded Markov points. Then we have that

$$
Q_{n+1}=\left\{\begin{array}{cc}
Q_{n}-1+A_{B}, & Q_{n} \geq 1 \\
A_{B}+A_{U}, & Q_{n}=0
\end{array}\right.
$$

where $A_{U}$ and $A_{B}$ be the numbers of data frames arriving during $U$ and $B$. From Eq. (2), we can give the P.G.F. $Q(z)$ and the average $E[Q]$ of $Q$ as follows:

$$
\begin{aligned}
& Q(z)=\frac{(1-\rho)(1-z) B(1-\lambda+\lambda z)}{B(1-\lambda+\lambda z)-z} \cdot \frac{1-z U(1-\lambda+\lambda z)}{(1+p E[U])(1-z)}, \\
& E[Q]=\rho+\frac{B^{(2)}}{2(1-\rho)}+\frac{2 \lambda E[U]+\lambda^{2} U^{(2)}}{2(1+\lambda E[U])}
\end{aligned}
$$

where $U^{(2)}$ and $B^{(2)}$ are the second factorial moments of $U(z)$ and $B(z)$. 
We denote by $W$ the waiting time of a data frame. Under the FIFO discipline, the number of data frames left in the system immediately after the transmission of a data frame is identical to the sum of the number of data frames arriving during the waiting time $W$ and the number of data frames arriving during the transmitting time $B$. Then the P.G.F. $Q(z)$ of $Q$ can also be written as follows:

$$
Q(z)=W(1-\lambda+\lambda z) B(1-\lambda+\lambda z) .
$$

By substituting $Q(z)$ of Eq. (3) to Eq. (4), we can obtain the P.G.F. $W(z)$ and the average $E[W]$ of $W$ as follows:

$$
\begin{aligned}
& W(z)=\frac{(1-\rho)(1-z)}{\lambda B(z)-z+1-\lambda} \cdot \frac{\lambda-(z-1+\lambda) U(z)}{(1+\lambda E[U])(1-z)}, \\
& E[W]=\frac{\lambda B^{(2)}}{2(1-\rho)}+\frac{2 E[U]+\lambda U^{(2)}}{2(1+\lambda E[U])}
\end{aligned}
$$

\subsection{Busy Period and Busy Cycle}

We define the busy cycle called $R$ as a time period from the instant in which a busy period is completed to the instant in which the next busy period ends. Let $E[R], E[\Theta]$ and $E[I]$ be the averages of the busy cycle $R$, the busy period $\Theta$ and the idle period $I$, respectively. We have that

$$
E[R]=E[U]+E[\Theta]+E[I]
$$

where $E[U]$ is defined in Eq. (1), and $E[\Theta]$ and $E[I]$ will be given below.

Each data frame at the beginning of a busy period $\Theta$ will introduce a subbusy period $\theta$. All $\theta$ brought by the data frames at the beginning of the busy period $\Theta$ combine to make a total busy period $\Theta$ in the system. Therefore, the P.G.F. $\Theta(z)$ and the average $E[\Theta]$ of $\Theta$ are given as follows:

$$
\Theta(z)=\theta(z) U(1-\lambda+\lambda \theta(z)), \quad E[\Theta]=\frac{(1+\lambda E[U]) E[B]}{(1-\rho)} .
$$

Considering the Bernoulli process, the average $E[I]$ of $I$ is given as follows:

$$
E[I]=\frac{1}{\lambda}
$$

Substituting Eqs. (7) and (8) to Eq. (6), the average $E[R]$ of $R$ is given by

$$
E[R]=\frac{1+\lambda E[U]}{\lambda(1-\rho)} .
$$

\section{Performance Measures for ARQ Schemes}

Based on the analysis above, we present some performance measures as follows.

Response time $T$ is defined as the total delay of a data frame. $T$ is subdividedinto two parts. One is the waiting time $W$ of this data frame, which is 
the time spent in the buffer before its transmission. The other is corresponding to the transmission period $B$ of this data frame. The average $E[T]$ of $T$ is given by

$$
E[T]=E[W]+E[B] .
$$

Next, we define utility $\eta$ as a ratio of the time period (one slot) in which a data frame is being transmitted correctly on a data link to the transmission period $B$ of the data frame. Clearly, the utility $\eta$ can be given by

$$
\eta=\frac{1}{E[B]} \text {. }
$$

To give the formulas for the performance measures of different kinds of ARQ schemes, the following assumptions and notions are introduced: (1) The transmissions of the ACK frame and the NACK frame are error free, and the lengths of the ACK frame and the NACK frame are omitted. (2) The rate of the transmission error is $e(0 \leq e \leq 1)$. Each data frame is correctly transmitted with probability $v=1-e(0 \leq v \leq 1)$, and each data frame will be transmitted or retransmitted until correct reception is achieved. (3) The round-trip-time is assumed to be $d$ slots as a system parameter.

Let $N$ be the number of times of transmission needed for a data frame to be received correctly. Then the probability distribution and the P.G.F. $N(z)$ of $N$ can be given as follows:

$$
P\{N=n\}=(1-v)^{n-1} v, \quad N(z)=\sum_{n=1}^{\infty} P\{N=n\} z^{n}=\frac{v z}{1-(1-v) z}
$$

where $n=1,2, \ldots$.

\subsection{Measures for Stop-and-Wait ARQ Scheme}

In the system with Stop-and-Wait ARQ scheme, each transmission will take $1+d$ slots, no matter whether the transmission is correct or not. From Eq. (12), we can obtain the P.G.F. $B_{S W}(z)$, the average $E\left[B_{S W}\right]$ and the second factorial moment $B_{S W}^{(2)}$ of the transmission period $B_{S W}$ for this case as follows:

$$
\begin{aligned}
& B_{S W}(z)=N\left(z^{1+d}\right)=\frac{v z^{1+d}}{1-(1-v) z^{1+d}}, \\
& E\left[B_{S W}\right]=\frac{1+d}{v}, \\
& B_{S W}^{(2)}=\frac{(1+d)(v d+2(1-v)(1+d))}{v^{2}} .
\end{aligned}
$$

Substituting Eqs. (14) and (15) to Eq. (10), we obtain the average response time $E\left[T_{S W}\right]$ as follows:

$$
\begin{aligned}
E\left[T_{S W}\right] & =E\left[W_{S W}\right]+E\left[B_{S W}\right] \\
& =\frac{\lambda\left((1+d)^{2}(2-v)+v d(1+d)\right)}{2(v-\lambda(1+d)) v}+\frac{2 E[U]+\lambda U^{(2)}}{2(1+\lambda E[U])}+\frac{1+d}{v}
\end{aligned}
$$

where the average waiting time $E\left[W_{S W}\right]$ of data frames is given by Eq. (5). 
Substituting Eq. (14) to Eq. (11), we give the utility ratio $\eta_{S W}$ as follows:

$$
\eta_{S W}=\frac{1}{E\left[B_{S W}\right]}=\frac{v}{1+d} .
$$

\subsection{Measures for Go-Back-N ARQ Scheme}

In the system with Go-Back-N ARQ scheme, each error transmission occupies $1+d$ slots, while the last correct transmission takes 1 slot. From Eq. (12), we can obtain the P.G.F. $B_{G B N}(z)$, the average $E\left[B_{G B N}\right]$ and the second factorial moment $B_{G B N}^{(2)}$ of the transmission period $B_{G B N}$ for this case as follows:

$$
\begin{aligned}
& B_{G B N}(z)=\frac{N\left(z^{1+d}\right)}{z^{d}}=\frac{v z}{1-(1-v) z^{1+d}}, \\
& E\left[B_{G B N}\right]=\frac{1+(1-v) d}{v}, \quad B_{G B N}^{(2)}=\frac{(1-v)(1+d)(2+2 d-v d)}{v^{2}} .
\end{aligned}
$$

By the same way, we can obtain the average response time $E\left[T_{G B N}\right]$ and the utility ratio $\eta_{G B N}$ for the Go-Back-N ARQ scheme as follows:

$$
\begin{aligned}
E\left[T_{G B N}\right] & =E\left[W_{G B N}\right]+E\left[B_{G B N}\right] \\
& =\frac{\lambda(1-v)(1+d)(2+2 d-v d)}{2(v-\lambda(1+(1-v) d)) v}+\frac{2 E[U]+\lambda U^{(2)}}{2(1+\lambda E[U])}+\frac{1+(1-v) d}{v}, \\
\eta_{G B N}= & \frac{1}{E\left[B_{G B N}\right]}=\frac{v}{1+(1-v) d} .
\end{aligned}
$$

where the average waiting time $E\left[W_{G B N}\right]$ of data frames is given by Eq. (5))

\subsection{Measures for Selective-Repeat ARQ Scheme}

In the system with Selective-Repeat ARQ scheme, each transmission, no matter whether it is correct or not, takes 1 slot. From Eq. (12), we can obtain the P.G.F. $B_{S R}(z)$, the average $E\left[B_{S R}\right]$ and the second factorial moment $B_{S R}^{(2)}$ of the transmission period $B_{S R}$ for this case as follows:

$$
B_{S R}(z)=N(z)=\frac{v z}{1-(1-v) z}, \quad E\left[B_{S R}\right]=\frac{1}{v}, \quad B_{S R}^{(2)}=\frac{2(1-v)}{v^{2}} .
$$

We can also give the average response time $E\left[T_{S R}\right]$ and the utility ratio $\eta_{S R}$ for the Selective-Repeat ARQ scheme as follows:

$$
\begin{aligned}
& E\left[T_{S R}\right]=E\left[W_{S R}\right]+E\left[B_{S R}\right]=\frac{\lambda(1-v)}{(v-\lambda) v}+\frac{2 E[U]+\lambda U^{(2)}}{2(1+\lambda E[U])}+\frac{1}{v}, \\
& \eta_{S R}=\frac{1}{E\left[B_{S R}\right]}=v
\end{aligned}
$$

where the average waiting time $E\left[W_{S R}\right]$ of data frames is given by Eq. (5). 


\section{$5 \quad$ Numerical Results}

In numerical results, considering the prevalent wireless network application, we let the transmission rate be $50 \mathrm{Mbps}$. To ensure the latest conflict signal be sensed by the transmitter before a data frame is completely sent out, we assume the size of a data frame to be 1250 bytes and the round-trip-time to be $0.1 \mathrm{~ms}$. The setup period $U$ is geometrically distributed with an average value of $0.2 \mathrm{~ms}$.

By using these parameters, we show the average response time $E[T]$ as a function of the arrival rate $\lambda$ (Data frames/Slot) with the rate of the transmission error $e=0.1$ and the utility ratio $\eta$ as a function of the rate of the transmission error for different kinds of ARQ schemes in Figs. 1, 2, where the schemes of the Stop-and-Wait ARQ, Go-Back-N ARQ and Selective-Repeat ARQ are abbreviated as SW ARQ, GBN ARQ and SR ARQ, respectively.

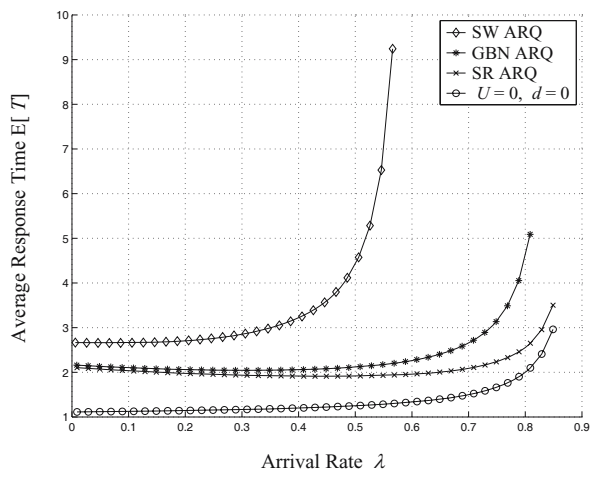

Fig. 1. Average response time $E[T]$

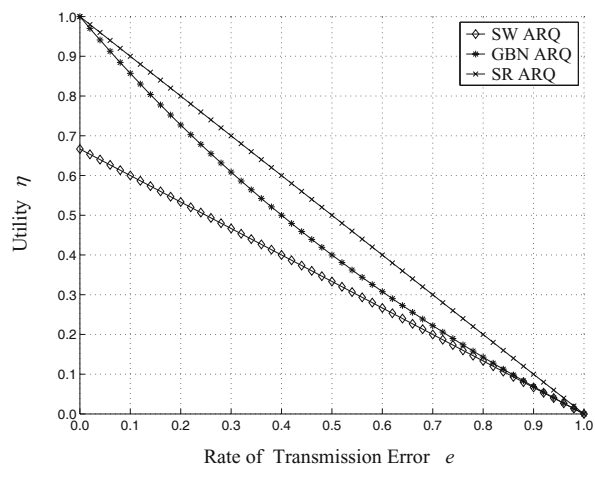

Fig. 2. Utility $\eta$

In Fig. 1, the curve of the lowest position shows the case without considering the delay of the setup procedure and the round-trip-time $(U=0$ and $d=0)$ for all the ARQ schemes. Namely, this case is that the setup procedure and the round-trip-time are not considered in the performance analysis and evaluation of the system as in previous papers [2], [3], [9].

Other curves of Fig. 1 are to show how the average response time $E[T]$ changes depend upon the delay of the setup procedure and the round-trip-time. It can be observed that when the arrival rate $\lambda$ is low, as $\lambda$ increases, there is a bit increase of $E[T]$ in Stop-and-Wait ARQ scheme, while there is a slight decrease for Go-Back-N ARQ scheme and Selective-Repeat ARQ scheme. This is because that the setup procedure for a data link has a heavier impact on Go-Back-N ARQ scheme and Selective-Repeat ARQ scheme than on Stop-and-Wait ARQ scheme. As $\lambda$ further increases, all the curves of these schemes increase suddenly, it is due to the heavy offered load $\rho$.

In Fig. 2, the utility ratio $\eta$ for all the ARQ schemes without considering the delay of the setup procedure and the round-trip-time $(U=0$ and $d=0)$ is just the same as the Selective-Repeat ARQ scheme. We can observe that the 
lower the rate of the transmission error $e$ is, the better the utility ratio $\eta$ will be for all the cases. We can also know that among these three schemes, the system performance of Selective-Repeat ARQ scheme is better than other two schemes. However, we also notice that when the rate of the transmission error $e$ is very high or very low, the utility $\eta$ for Go-Back-N ARQ scheme and for Selective-Repeat ARQ scheme tends to be the same.

\section{Conclusions}

Performance analysis of high-reliability Internet systems in wireless environments with ARQ schemes was presented. Considering the setting up procedure of a data link, we built a Geom/G/1 queue model with a setup strategy to characterize the system operation. Taking into account the round-trip-time, we analyzed the stationary distribution of the system and gave the formulas of performance measures for different kinds of ARQ schemes. We presented numerical results to evaluate and compare the performance of the systems with different kinds of ARQ schemes in terms of the average response time of data frames and the utility ratio of the system, and to show influence of the delay of the setting up procedure and the round-trip-time on the system performance.

Acknowledgments. This work was supported in part by MEXT.ORC (20042008), Japan and in part by NSFC (No. 10671170) and MADIS, China.

\section{References}

1. Yue W. and Matsumoto Y.: Performance Analysis of Multi-Channel and MultiTraffic on Wireless Communication Networks. Kluwer Academic Publishers (2002)

2. Badia L., Rossi M. and Zorzi M.: SR ARQ Packet Delay Statistics on Markov Channels in the Presence of Variable Arrival Rate. IEEE Transaction on Wireless Communication. 5 (2006) 1639-1644

3. Zheng H. and Viswanthan H.: Optimizing the ARQ Performance in Downlink Packet Data Systems With Scheduling. IEEE Transaction on Wireless Communication. 4 (2005) 495-506

4. Takagi H.: Queueing Analysis. Vol. 3: Discrete-Time Systems. North-Holland (1993)

5. Tian N. and Zhang G.: Vacation Queueing Models-Theory and Applications. Springer-Verlag (2006)

6. Tian N. and Zhang G.: The Discrete Time GI/Geo/1 Queue With Multiple Vacations. Queueing Systems. 40 (2002) 283-294

7. Jin S. and Tian N.: Performance Evaluation of Virtual Channel Switching System Based on Discrete Time Queue. Journal of China Institute of Communications. 25 (2004) 58-68 (in Chinese)

8. Jin S., Yue W. and Liu M.: Performance Analysis of SVC Based on Discrete Time Factorization Principle With General Vacations. Technical Report of IEICE. 102 (2005) 1-6

9. Hou F., Ho P., Xue M. and Zhang Y.: Performance Analysis of Differentiated ARQ Scheme for Video Transmission over Wireless Networks. International Workshop on Modeling Analysis and Simulation of Wireless and Mobile Systems (2005) 1-7 